\title{
Método Mãe Canguru: o cuidado compartilhado com a atenção primária em saúde
}

Kangaroo mother method: shared care whit primary health care

\section{Método madre canguro: atención compartida con atención primaria de salud}

\section{Ana Carolina Alvim Pessoa Alves ${ }^{1 *}$, Alessandra da Silva Souza ${ }^{2}$, Jannaina Sther Leite Godinho Silva ${ }^{3}$, Manoela Alves ${ }^{4}$, Eliara Adelino da Silva ${ }^{5}$, Elisangela Nascimento Fernandes Gomes ${ }^{6}$}

Como citar esse artigo. Alves ACAP; Souza, AS; Silva, JSLG; Alves, M; Silva, EA; Gomes, ENF. Método Mãe Canguru: o cuidado compartilhado com a atenção primária em saúde. Revista Pró-UniverSUS. 2021 Jul./Dez.; 12 (2): $67-71$

\section{Resumo}

O Método Mãe Canguru (MMC) surgiu inicialmente na Colômbia com a proposta de melhorar os cuidados prestados ao recém nascido pré termo, tendo como intuito reduzir os custos da assistência perinatal e promover, através do contato pele a pele precoce entre a mãe e o seu bebê, maior vínculo afetivo, maior estabilidade térmica e melhor desenvolvimento. Nesta perspectiva, devido ao vazio literário de materiais detalhados, o presente estudo tem por objetivo identificar como se dá o cuidado ao recém-nascido pré-termo e sua família após a alta hospitalar, bem como seus desafios e dificuldades. Para tal, foi realizado uma revisão bibliográfica com abordagem qualitativa utilizando-se da busca de artigos científicos, guias e protocolos para expansão do conhecimento. Durante a revisão foram identificados aspectos relevantes a serem abordados, como: o preparo para a continuidade do método mãe canguru; a interface da $3^{\mathrm{a}}$ etapa com a atenção básica; o enfermeiro como incentivador do método mãe canguru na atenção básica.Sabe-se que, inicialmente, as ações do MMC são centradas no cuidado hospitalar, no entanto, gradativamente há a construção de uma linha de cuidado que integra o trabalho da Unidade Neonatal à Atenção Básica, as quais devem ser grandes aliadas. Sendo assim, para que a assistência na APS seja efetiva, faz-se necessário que os profissionais sejam sensibilizados e capacitados para realizar as ações necessárias, por meio de uma assistência articulada que inclui os diversos níveis de saúde.

Palavras-chave: Método Mãe Canguru; Terceira Etapa; APS.

\begin{abstract}
The Kangaroo Mother Method (MMC) emerged in Colombia with the proposal to improve the care provided to preterm newborns, with the aim of reducing the costs of perinatal care and promoting, through early skin-to-skin contact between the mother and her baby, greater affective bond, greater thermal stability and better development. In this perspective, due to the literary emptiness of detailed materials, the present study aims to identify how the preterm newborn and his family are cared for after hospital discharge, as well as their challenges and difficulties. To this end, a bibliographic review with a qualitative approach was carried out using the search for scientific articles, guides and protocols for expanding knowledge. During a review, the relevant aspects to be separated were identified, such as: the preparation for the continuity of the kangaroo mother method; the interface of the 3rd stage with primary care; the nurse as a promoter of the kangaroo mother method in primary care. It is known that, in a bulletin, MMC's actions are centered on hospital care, however, gradually there is the construction of a line of care that integrates the work of the Neonatal Unit with Primary Care, such as which should be great allies. Therefore, in order for PHC assistance to be effective, it is necessary that professionals are sensitized and trained to carry out the necessary actions, through articulated assistance that includes the different levels of health.
\end{abstract}

Keywords: Kangaroo Mother Method; Third Stage; PHS.

${ }^{2}$ Mestre. Docente do curso de Enfermagem. Universidade de Vassouras, Vassouras, RJ. Brasil. E-mail:alesouza22@yahoo.com.br ORCID: https://orcid.org/0000-0002-9009-9774

${ }^{3}$ Mestre. Docente do curso de Enfermagem. Universidade de Vassouras, Vassouras, RJ. Brasil. E-mail: jjasther@gmail.com ORCID: https://orcid.org/0000-0002-8308-2093

${ }^{4}$ Mestre. Docente do curso de Enfermagem. Universidade de Vassouras, Vassouras, RJ. Brasil. E-mail: alves.manoela@gmail.comORCID: https://orcid.org/0000-0003-4239-9577

${ }^{5}$ Mestre. Docente do curso de Enfermagem. Universidade de Vassouras, Vassouras, RJ. Brasil. E-mail: eliaraadelinos@uol.com.brORCID: https://orcid.org/0000-0003-4387-6662

${ }^{6}$ Mestre. Docente do curso de Enfermagem. Universidade de Vassouras, Vassouras, RJ. Brasil. E-mail: elisangelavass@yahoo.com.brORCID: https://orcid.org/0000-0001-8432-4157 


\section{Resumen}

El Método Madre Canguro (MMC) surgió en Colombia con la propuesta de mejorar la atención brindada a los recién nacidos prematuros, con el objetivo de reducir los costos del cuidado perinatal y promover, a través del contacto temprano piel a piel entre la madre y su bebé, mayor vínculo afectivo, mayor estabilidad térmica y mejor desarrollo. En esta perspectiva, debido al vacío literario de materiales detallados, el presente estudio tiene como objetivo identificar cómo se atiende al recién nacido prematuro y su familia después del alta hospitalaria, así como sus desafíos y dificultades. Para ello, se realizó una revisión bibliográfica con enfoque cualitativo mediante la búsqueda de artículos científicos, guías y protocolos para la expansión del conocimiento. Durante una revisión se identificaron los aspectos relevantes a separar, tales como: la preparación para la continuación del método madre canguro; la interfaz de la $3^{\mathrm{a}}$ etapa con la atención primaria; la enfermera como promotora del método madre canguro en atención primaria. Se sabe que, en un boletín, las acciones de MMC se centran en la atención hospitalaria, sin embargo, paulatinamente se va construyendo una línea de atención que integra el trabajo de la Unidad Neonatal con la Atención Primaria, por lo que deben ser grandes aliados. Por lo tanto, para que la asistencia en la APS sea efectiva, es necesario que los profesionales estén sensibilizados y capacitados para realizar las acciones necesarias, a través de una asistencia articulada que incluya los diferentes niveles de salud.

Palabras clave: Método Madre Canguro; Tercera etapa; APS.

\section{Introdução}

A ideia de que a própria mãe pudesse ser a fonte de calor para seu bebê prematuro surgiu na Colômbia em 1985 descrito por WhitelaweSleath, devido à escassez de incubadoras. No Brasil, o Ministério da Saúde (MS) ${ }^{1}$, lançou a Norma de Orientação para a Implementação do Método Canguru, a qual estabelece as diretrizes para sua aplicação nas unidades do Sistema Único de Saúde (SUS). Em geral, ela prevê:abertura das Unidades Neonatais (UN) para que os pais possam tocar o(a) filho(a) o mais rápido possível; contato pele a pele com a mãe, favorecendo o bem-estar e a adaptação mais rápida do bebê à vida extra-uterina, bem como estimular a prática da amamentação; alta hospitalar precoce e continuidade do contato pele a pele no domicílio.

Sendo assim, o Método Mãe Canguru (MMC) é considerado um modelo de assistência ao recémnascido pré-termo (RNPT) e sua família, internado na Unidade de Tratamento Intensivo Neonatal, voltado para o cuidado humanizado, que reúne estratégias de intervenção biopsicossocial.

O peso dos prematuros, nos primeiros dias de vida e internação, sofre influências tanto do seu quadro clínico instável, como dos estressores ambientais aos quais estes recém nascidos (RN) são submetidos, ocasionando perda de peso característica ${ }^{2}$. No Método Mãe-Canguru, na proporção em que o RN fica clinicamente estável e inicia um processo lento de ganho ponderal, passa ser intensificado seu contato com a mãe, em termos de tempo e qualidade.

O MMC foi desenvolvido para estimular as competências do Aleitamento Materno Exclusivo (AME), proporcionando maior frequência, periodicidade e duração com livre demanda da amamentação, única fonte de nutrição e proteção nos primeiros seis meses de vida. Por conseguinte, o AME em RNPT diminui o uso de suplementação alimentar para os bebês e a melhora da produção láctea materna. ${ }^{3}$
De acordo com algumas literaturas, acredita-se que o MMC pode influenciar nas variáveis respiratórias, evidenciado pela estabilidade da caixa torácica e alterações nas funções hemodinâmicas do prematuro, conseguido através do contato pele a pele com a mãe, este que fornece adequado posicionamento. E ainda, reduz os números de internações, reduz infecções hospitalares e consequentemente, o índice de morbimortalidade do RNPT. Ajuda também na passagem mais rápida da incubadora ao berço com a alta antecipada. ${ }^{4}$

Sabe-se então que o MMC é realizado em três etapas, a primeira na Unidade de Terapia Intensiva Neonatal (UTIN) e na Unidade de Cuidado Intermediário Neonatal Convencional (UCINCo), a segunda na Unidade de Cuidado Intermediário Neonatal Canguru (UCINCa) e a terceira após a alta hospitalar, no domicílio. ${ }^{5}$

A partir destas discussões, visto que já são diversas as pesquisas quanto a internação e que, existe um vazio literário de artigos detalhados sobre a $3^{\text {a }}$ etapa do MMC, este estudo visa identificar o cuidado e seus desafios ao recém-nascido pré-termo e sua família após a alta hospitalar, com enfoque na atuação do enfermeiro, de formaa garantir melhoria na assistência prestada.

\section{Materiais e métodos}

Tratou-se de uma revisão bibliográfica com abordagem qualitativa baseada na revisão literária de artigos científicos a partir da busca pelas bases de dados Scientific Eletonic Library Online(Scielo), Literatura Latino Americana e do Caribe em Ciência da Saúde (Lilacs)e Base de dados de Enfermagem (Bdenf). A coleta dos artigos foi realizada no período correspondente a setembro de 2020 a dezembro de 2020. Os descritores utilizados para pesquisa foram: Método Canguru; Terceira Etapa.

Inicialmente, fez-se a leitura dos resumos 
encontrados com intuito de identificar a pertinência ao objeto em estudo, incluindo os artigos em idioma português, relacionados a enfermagem, sem delimitação temporal devido à escassez de pesquisas, sendo então encontrados 12 artigos. Posteriormente, foram selecionados 03 artigos, os quais atendiam ao critério, sendo excluído os demais. Foi utilizado também a apreciação de guias e protocolos nacionais condizentes ao tema.

"Pesquisa qualitativa é o que se aplica ao estudo da história, relações e interpretações, crenças, opiniões e percepções do ser humano permiti desvendar os processos sociais para análises de discursos e documentos." 6:21-22

\section{Resultados e Discussão}

Durante a revisão foram identificados alguns aspectos relevantes no processo de análise para construção do estudo. Desta forma, as pesquisas foram agrupadas refletindo o objetivo geral em cada eixo temático: o preparo para a continuidade do método mãe canguru; a interface da $3^{\text {a }}$ etapa com a atenção básica; o enfermeiro como incentivador do método mãe canguru na atenção básica.

\section{O preparo para a continuidade do método mãe canguru}

O preparo para a continuidade do MMC engloba desde o recém-nascido, a família, até os profissionais de saúde da atenção hospitalar, da atenção especializada e da atenção básica. Antes da alta hospitalar, ainda na $2^{\mathrm{a}}$ etapa, já deve ser iniciado o contato entre a equipe do hospital e a da Atenção Básica (AB), este contato irá possibilitar a troca de informações trazendo vantagens para o RNPT e sua família e permitindo um cuidado integral por meio da intersetorialidade dessas equipes. ${ }^{7}$

É importante lembrar que, a ida para a casa requer um olhar especial e diferenciado, pois a criança e sua família irão passar por novas experiências e sensações. Tudo mudará, cheiros, barulhos, vozes, temperaturas. Portanto, cabe aos profissionais prepará-los para que deem conta das novidades.

Neste cenário, o principal objetivo é observar as possibilidades que a criança passa a mostrar de permanecer no lar sob os cuidados dos responsáveis. Ela irá receber alta hospitalar, mas com a garantia de que estará assegurada para retorno caso surja essa necessidade. ${ }^{5}$

Ainda na $2^{\mathrm{a}}$ etapa, a família deve ser orientada e estimulada a realizar o acompanhamento na Atenção Primária a Saúde (APS) para dar continuidade ao MMC durante a $3^{\circ}$ etapa. ${ }^{7,8}$ Porém, percebe-se que há fragilidade nas orientações que dizem respeito ao acompanhamento da criança após a alta. Como consequência da comunicação falha, o que acontece, é que eles acabam procurando o pronto socorro hospitalar como porta de entrada.

\section{A interface da $3^{a}$ etapa com a atenção básica}

Pesquisas apontam que um dos principais desafios da articulação do cuidado especializado com a atenção básica é a percepção que as crianças vindas da Unidade Neonatal são frágeis e imaturas fisiologicamente. Percebe-se também, diante de estudos já realizados, que boa parte dos profissionais de saúde não assimilam que o bebê nascido pré-termo tem as necessidades básicas de qualquer outro, sendo estas muitas vezes subestimadas pelo medo e insegurança na prestação da assistência. ${ }^{7}$

Na maternidade, a equipe responsável pela terceira etapa realiza consultas programadas e de demanda espontânea. E ao mesmo tempo, a criança começa a ser assistida pela equipe da APS e a receber visitas.

Até que o recém-nascido atinja $2.500 \mathrm{~g}$, é recomendado que ele passe por três consultas na primeira semana, duas na segunda semana e a partir da terceira semana, consultas semanais. O MS propõe que os profissionais da $\mathrm{AB}$ participem efetivamente da terceira etapa do MMC, através de consultas e visita domiciliar, mas isto ainda não é a realidade na maioria dos municípios brasileiros. ${ }^{5}$

O bebê pré-termo na atenção básica tem que ser acompanhado utilizando-se as curvas sugeridas pela OMS, fazendo-se necessário que a idade seja ajustada de acordo com o tempo de prematuridade. A avaliação correta deve ser feita corrigindo a idade do bebê para peso e estatura até 2 anos e perímetro cefálico até 18 meses. $^{5}$

Nesta perspectiva, é recomendado pelo MS que o RNPT seja acompanhado tanto na atenção especializada quanto na atenção primária. Isso permite o compartilhamento dos dois níveis de atenção e auxilia na melhoria do cuidado continuado.

As equipes que compartilham a terceira etapa devem estar sempre atentas e compartilhando cuidados que envolvem por exemplo: manutenção da posição canguru, amamentação, seguimento das consultas, vacinação, realização de exames, entre outros. Porém, este cuidado não se mostra presente na APS, deixando perceptível a dificuldade de entender que a criança de risco é responsabilidade de toda a rede de serviços de saúde.

Em outro cenário, foi possível perceber que parte da dificuldade de manter o cuidado continuado na $\mathrm{AB}$ tem relação com o conhecimento limitado que os profissionais possuem sobre a terceira etapa do MMC, fazendo com que se não se sintam aptos para tal. Isto é evidenciado pela comunicação falha entre o hospital e a Unidade de Saúde. Portanto, para que haja a real 
efetivação da terceira etapa, é necessário capacitações e pactuações entre os níveis de saúde. Vale ressaltar, que esta questão já foi apontada pelo MS através da Política de Atenção Integral a Saúde da Criança. ${ }^{8}$

$\mathrm{O}$ suporte e as orientações dadas pela equipe de saúde são fatores determinantes para que se tenha sucesso do MMC no domicílio, visto a complexidade quanto a transição do setor hospitalar para a residência do recém-nascido.

\section{$O$ enfermeiro como incentivador do método mãe canguru na atenção básica}

O enfermeiro da atenção básica é essencial no processo de alta hospitalar e seguimento do MC no domicílio, ele poderá auxiliar, ajudar e orientar a mãe, o RN e sua família para que este processo seja o menos complicado possível. Sendo assim, um dos primeiros passos do enfermeiro deverá ser solicitar o relatório de alta do RNPT com dados da criança desde o seu nascimento até a alta hospitalar, buscando conhecer e entender sobre o caso. Neste relatório constará algumas informações básicas do bebê, como: aleitamento materno e alimentação suplementar, exames, agendamentos, doenças e problemas, peso, perímetro cefálico e comprimento, medicações e necessidade de acompanhamento especializado. ${ }^{9}$

Para o cuidado efetivo, integral e humanizado, a equipe da Atenção Básica deve também realizar visitas domiciliares, sendo recomendado que esta ocorra pelo menos uma vez por semana durante toda terceira etapa. Sendo a visita domiciliar, um instrumento valioso que facilita a compreensãodas redes sociofamiliares, seu modo de vida, cultura, crenças e padrões de comportamento. ${ }^{9}$

Além disso, as visitas permitirão que o enfermeiro e a sua equipe identifiquem situações de risco e dinâmica familiar, acompanhem aleitamento materno e ganho de peso, forneça orientações quanto vacinação, consultas, pega correta e realize a busca ativa de crianças que faltaram às consultas.

Outro aspecto importante que o enfermeiro deve observar é em relação ao aleitamento materno associado ao ganho de peso. Estudos comprovam que a falta de orientação, pega incorreta, medo associado a prematuridade e insegurança das mães, é umas das principais causas da dificuldade encontrada para a prática do AME. Sendo possível perceber, que a maioria destes problemas acontecem por falhas que podem ser corrigidas com acompanhamento e orientações corretase contínuas, seguidas de ações educativas sobre aleitamento. ${ }^{10}$

Entre todas as orientações que o enfermeiro deve dar a família durante as visitas, pode-se destacar quatro de maior relevância, sendo elas: posicionamento do bebê, que irá propiciar conforto e segurança, sendo a recomendada a posição canguru, e orientar que no berço o bebê deve ser colocado de barriga para cima, pois previne a morte súbita;controle da temperatura, lembrando que a posição canguru proporciona um bom controle térmico; observação das eliminações, perguntando sobre quantidade, aspecto, frequência e cheiro; orientações quanto a banho e higiene, explicando que este é um momento de interação, sendo importante que o pai também participe. ${ }^{9}$

É importante destacar também sobre os prematuros que são mais vulneráveis a apresentarem sequelas, necessitando de cuidados especializados, são alguns deles: baixo peso extremo; anóxia perinatal grave; problemas respiratórios como a doença de membrana hialina e displasia broncopulmonar; problemas neurológicos como acidente vascular cerebral, convulsão e sangramento de sistema nervoso central; malformações e síndromes genéticas, entre outros. $\mathrm{O}$ objetivo do seguimento à essas crianças advindas da UTIN é intervir, prevenir, detectar ou minimizar alterações e complicações, bem como dar apoio, suporte e fornecer orientações a família e o bebê. Para que este objetivo seja cumprido, os profissionais envolvidos nesta assistência, incluindo o enfermeiro da $\mathrm{AB}$, devem fornecer aos pais capacitação e conhecimento adequados e corretos referente aos cuidados especiais com seus filhos, como por exemplo: alimentação por gavagem; cuidados com estomias; medicações; banho e higiene; oxigenoterapia domiciliar; reconhecimento de sinais de alerta e procedimentos que poderão ser realizados nestas situações. ${ }^{11} \mathrm{E}$ além disto, o enfermeiro através das visitas deverá e poderá avaliar os recursos domiciliares, buscando assegurar que o bebê e sua família estão tendo todo suporte necessário.

\section{Considerações Finais}

Foi identificado que grande parte do cuidado descontinuado e negligenciado ao RNPT e sua família na APS, tem relação direta com a falta de conhecimento, estímulo e comunicação entre os serviços de saúde.

A interdisciplinaridade fortifica o conhecimento e a experiência profissional e pessoal, fazendo com que o compartilhamento de vivências e saberes resulte no cuidado integral, acolhedor e humanizado. Durante o MMC, principalmente durante a $3^{\mathrm{a}}$ etapa, este compartilhamento se faz fundamental, visto que, deve acontecer a articulação entre a atenção especializada e a APS, mais especificamente a Unidade de Saúde referente ao domicílio da família assistida.

Logo, mostra-se necessária a aprimoração do conhecimento e desmistificações através de estratégias de educação permanente e capacitações para os profissionais de saúde de todos os níveis de atenção. Bem como, é necessário que a abordagem seja baseada 
na integralidade do cuidado e na integração da equipecriança-família. O cuidado prestado deve ser contínuo e iniciado o mais precocemente possível, podendo assim minimizar as dificuldades encontradas neste processo. Para tal, os serviços de saúde devem compreender a família e a criança como uma unidade, ou seja, devem entender seu funcionamento, seus recursos e vulnerabilidades.

Cabe ressaltar que houve limitação na captação de dados para o estudo, uma vez que, o tema proposto ainda possui poucas evidências do seu uso na prática. Contudo, o objetivo inicial foi alcançado dentro das possibilidades.Apesarda evoluçãohistórica sobre a saúde da criança ainda existem lacunas no cuidado do recémnascido de baixo peso entre os eixos organizacionais no fortalecimento das políticas públicas.

\section{Referências}

1. Brasil. Ministério da Saúde. Portaria $n^{\circ}$ 693, de 5 de julho de 2000. Norma de orientação para implementação do Método Mãe Canguru. Diário Oficial da União. 06 de jul de 2000; Seção 1:129,

2. Toma TS. Método Mãe Canguru: o papel dos serviços de saúde e das redes familiares no sucesso do programa. Cad. Saúde Pública, Rio de Janeiro. 2003; 19 (2): S233-S242,

3. Costa R, Monticelli M. Método Mãe-Canguru. Acta paul. Enferm, 2005; 18 (4): 427-432.

4. Tenório EAM et al. Avaliação dos parâmetros fisiológicos em recémnascidos pré-termos de baixo peso antes e após a aplicação do método mãecanguru. Fisioterapia Brasil. jan./fev. 2010; 11 (1): 44 -47.

5. Brasil. Ministério da Saúde. Atenção Humanizada ao Recém-Nascido de Baixo Peso. Método Canguru, Manual Técnico - $2^{\circ}$ edição, Brasília DF, 2011

6. Minayo, MC de L (Org.) Pesquisa social: teoria, método e criatividade. 19. Petrópolis: Vozes, 2014.

7. Silva MS, Lamy ZC, Simões VMF, Pereira MCL, Campelo CMC, Gonçalves LLM. Acompanhamento na terceira etapa do método canguru: desafios na articulação de dois níveis de atenção. Revista Baiana de Saúde Pública. out./dez. 2018; 42 (4): 671-685.

8. Aires LCP, Santos EKA, Costa R, Borck M, Custódio ZAO. Seguimento do bebê na atenção básica: interface com a terceira etapa do método canguru. Rev. Gaúcha Enferm. 2015; 36 (spe): 224-232.

9. Brasil. Ministério da Saúde. Secretaria de Atenção à Saúde. Departamento de Ações Programáticas Estratégicas. Guia de orientações para o Método Canguru na Atenção Básica: cuidado compartilhado. Brasília, 2016.

10. Vasconcelos TC, Barbosa DJ, Gomes MP. Fatores que interferem no aleitamento materno exclusivo durante os primeiros seis meses de vida do bebê. Revista Pró-UniverSUS. 2020 Jan./Jun.; 11 (1): 80-87.

11. Viera CS, Mello DF. O seguimento da saúde da criança pré-termo e de baixo peso egressa da terapia intensiva neonatal. Texto contexto - enferm. 2009; 18(1): 74-82. 